\title{
Isolation, Identification, and Characterization of the Novel Antibacterial Agent Methoxyphenyl-Oxime from Streptomyces pratensis QUBC97 Isolate
}

\author{
Barghouthi SA ${ }^{* 1}$, Ayyad I ${ }^{1}$, Ayesh $\mathrm{M}^{1}$, and Abu-Lafi S ${ }^{2}$ \\ ${ }^{1}$ Medical Laboratory Sciences, Faculty of Health Professions, Jerusalem, Palestine \\ ${ }^{2}$ Faculty of Pharmacy, Al-Quds University, Jerusalem, Palestine
}

${ }^{*}$ Corresponding author: Barghouthi SA, Medical Laboratory Sciences, Faculty of Health Professions, Jerusalem, Palestine, Fax: +972-2-2791234, Tel: +972-599-379561, E-mail: bargsam@yahoo.com

Citation: Barghouthi SA, Ayyad I, Ayesh M, Abu-Lafi S (2017) Isolation, Identification, and Characterization of the Novel Antibacterial Agent Methoxyphenyl-Oxime from Streptomyces pratensis QUBC97 Isolate. J Antibio Res 1(1): 105. doi: 10.15744/2574-5980.1.105

Received Date: January 11, 2017 Accepted Date: February 28, 2017 Published Date: March 03, 2017

\begin{abstract}
Introduction: Streptomyces species are medically, industrially, and microbiologically important bacteria that are found in soils and other habitats.

Methods: Streptomyces isolates and QUBC97 were cultured on a new medium (CCG). Agar plug diffusion test showed antibacterial activity. N-butanol extracts analysed by silica thin layer chromatography (TLC), HPLC purified, and identified by MS analysis. QUBC97 was identified based on morphology, PCR, and DNA sequencing of two loci.

Results: CCG medium allowed Streptomyces isolation, growth, and antibacterial agent production. QUBC97 was identified as Streptomyces pratensis. In CCG broth, it produced two distinguishable antibacterial compounds; a one-week fresh compound (FCm) or a 6-week old compound (OCm). Agar cultures showed a mixture of both compounds. Split peak absorptions (426/442 nm) was observed for FCm and ( $428 / 442 \mathrm{~nm}$ ) for OCm. TLC developed in n-butanol saturated with ammonium acetate showed Rf, 0.86 for FCm and 0.67 for OCm. Mass-spectroscopy of HPLC pure FCm, identified FCm as methoxyphenyl-oxime. Both FCm and OCm were active against bacteria. FCm showed activity against a number of Gram negative and Gram positive clinical bacterial isolates.

Conclusion: CCG medium, agar plug test, and TLC bioassay formed a simple effective system for evaluation of antimicrobial activity. This is likely applicable to other extracts. Coupling molecular and phenotypic characteristics must be applied in Streptomyces species identification. Methoxyphenyl-oxime appeared to have a selective antibacterial activity since some bacterial isolates were resistant as indicated by the disc diffusion method.
\end{abstract}

Keywords: Streptomyces pratensis; TLC bioassay; Methoxyphenyl-oxime; CCG medium; Agar plug assay; Antibacterial agents; Antibiotic

\section{Importance}

Bacterial resistance to antibiotics is on the rise, both at the qualitative and quantitative levels. Searching for new antibiotics is an ongoing race in controlling pathogens, especially pathogens that have acquired antibiotic resistance. Our research may significantly contribute to finding new antibiotics. Our work has simplified and improved research tools and methods used for screening byproducts of Streptomyces spp. (or other sources such as plant extracts, fungi, Bacillus spp., and synthetic compound) for antibiotics or other bioactive agents. Our work may serve as a model for similar studies. Methoxyphenyl, oxime (MPO) may prove to be a therapeutic antibacterial agent that is effective against resistant bacteria such as the methicillin resistant staphylococcus aureus (MRSA). MPO may be prepared at commercial scale, synthesized, and modified to enhance its properties.

\section{Introduction}

Streptomyces flavogriseus IAF-45-CD (ATCC 33331) was reclassified as Streptomyces pratensis. Its DNA codes for carbapenem and a $\beta$-lactamase inhibitor [1].

Identification of Streptomyces species and the bioactive compound(s) they produce are complex and problematic [1-4]. Major obstacles in identifying Streptomyces species include horizontal gene transfer, erroneous reporting of species [5], recombination, chromosome rearrangement that may take place in Streptomyces [6,7], and replacing the scientific species name with a number 
(e.g. Streptomyces sp. Xxxx) which increases uncertainty of species identity. Discrepancies between 16S based phylogrouping and multilocus based phylogrouping suggest that accurate species identification is difficult [5]. Sequences of $16 \mathrm{~S}$ of Streptomyces phylogroup pratensis are identical for eight species in the S. griseus clade. Such diverse identity (calculated by multiplying BLAST sequence coverage by its percent BLAST identity) showing genomic variations between species of the genus Streptomyces to range from 20.4 to $100 \%$ in this example (Table 1). A practical system to reclassify Streptomyces species may be necessary.

\begin{tabular}{|c|c|c|c|c|}
\hline Streptomyces Species & $\begin{array}{c}\% \text { coverage } \\
\text { (A) }\end{array}$ & $\begin{array}{c}\% \text { identity } \\
(\mathbf{B})\end{array}$ & $\begin{array}{c}\text { Total identity } \\
(\mathbf{T i}) *(\mathbf{A} \times \mathbf{B}) \%\end{array}$ & $\begin{array}{c}\text { Accession } \\
\text { Number }\end{array}$ \\
\hline S. griseus subsp. griseus NBRC 13350 & $100 \%$ & $100 \%$ & 100 & AP009493.1 \\
\hline S. globisporus C-1027 & $70 \%$ & $94 \%$ & 66 & CP013738.1 \\
\hline S. fulvissimus DSM 40593 & $67 \%$ & $92 \%$ & 62 & CP005080.1 \\
\hline S. pratensis ATCC 33331 & $51 \%$ & $88 \%$ & 45 & CP002475.1 \\
\hline Streptomyces sp. PAMC26508 & $51 \%$ & $88 \%$ & 45 & CP003990.1 \\
\hline Streptomyces sp. SirexAA-E & $50 \%$ & $88 \%$ & 44 & CP002993.1 \\
\hline Streptomyces sp. CCM_MD2014 & $36 \%$ & $91 \%$ & 33 & CP009754.1 \\
\hline S. albulus strain NK660 & $31 \%$ & $90 \%$ & 27.9 & CP015726.1 \\
\hline S. cattleya str. NRRL 8057 & $30 \%$ & $92 \%$ & 27.6 & CP007574.1 \\
\hline S. xiamenensis strain 318 & $24 \%$ & $85 \%$ & 20.4 & FQ859185.1 \\
\hline
\end{tabular}

${ }^{*}$ Total identity $(\mathrm{Ti})=\mathrm{A} \times \mathrm{B}$; exact identity $=100 \%$, if $\mathrm{A}=50 \%$ and $\mathrm{B}=90 \%$, then $\mathrm{Ti}=45 \%$

Table 1: BLAST results with genome of Streptomyces griseus subsp. Griseu. Accession|AP009493.1| showing a huge range

of variation among a short list of selected species of the genus Streptomyces indicating the high diversity of the genus

We suggest the creation of a "Hybrid Bank of Taxonomy" connecting morphological features to DNA sequences such as 16S and/or other sequences; i.e. Streptomyces grouped based on $16 \mathrm{~S}$ similarity must then be distinguished based on morphological, molecular features, signature protein(s), DNA "tags", and/or distinguishable character of a species may provide such a simple system of species identification [this work, 3-5, 8-10]. Taddei et al. [3] applied morphological and biochemical tests to differentiate between 71 isolates obtained from Venezuelan soils. The tests failed to confirm or rule out identity [3]. Ju et al. identified gene clusters of phosphonic acid metabolism among 10,000 actinomycetes genomes. They classified gene clusters into 64 clusters encompassing 278 strains. These clusters may represent important tags for such strains allowing their separation from other actinomycetes. They also identified eleven undescribed compounds one of which is phosphonocystoximate [4]. Gao and Gupta discussed "The Molecular Signatures" for Actinobacteria. The concatenated protein sequences represent molecular signature proteins placing sequenced Streptomyces spp. in a single cluster [8]. Rong et al. utilized five concatenated DNA sequences to construct a phylogenetic tree that was different from the 16S-phylogenetic tree of the same isolates [5]. Shirling and Gottlibe indicated that their goal was to identify stable taxonomical properties of Streptomyces spp. [9]. Seventy years ago, Waksman and Lechevalier published their manual for Streptomyces classification and identification [10].

Such a bank of information will allow systematic analyses of all species and their by-products if we are to fight multidrug resistant microbes and cancer.

In this work, we report the isolation of the antibacterial agent methoxyphenyl-oxime as identified by MS analysis from a Streptomyces isolate QUBC97 which was closely related to Streptomyces phylogroup pratensis as suggested by phenotypic, multilocus, and DNA sequence analyses.

\section{Materials and Methods}

\section{CCG culture medium and isolation of Streptomyces}

Unrelated soil samples (200-300 g) were collected from a depth of $5 \mathrm{~cm}$ in fresh plastic bags and stored in wooden drawers at room temperature. CCG selective agar medium ( $0.1 \%$ casamino acids proteolytic hydrolysate, Sigma Chemicals; $0.1 \%$ trisodium citrate; $2 \%$ agar made in Jericho ${ }^{\star}$ bottled water as a source of trace elements, $\mathrm{pH} \sim 7.8$ before autoclaving;). Autoclaved media (broth or agar) were cooled to $\sim 50{ }^{\circ} \mathrm{C}$; autoclaved glycerol ( $50 \% \mathrm{v} / \mathrm{v}$ made in water) was aseptically added to a final concentration of $0.5 \%$ v/v. Filter sterilized cycloheximide at $100 \mu \mathrm{g} / \mathrm{ml}$ was added to CCG medium to make CCCG medium which was used occasionally to control contaminating fungi. CCG cultures were used to evaluate bioactivities of Streptomyces spp. Nutrient agar from BD Difco, USA was used in disc diffusion tests and the initial isolation of isolate QUBC97 from a commercial cacao sample, the isolate was transferred to CCG medium.

Primary Streptomyces agar cultures $\left(28{ }^{\circ} \mathrm{C}\right)$ were microscopically inspected for colonies $(4 \mathrm{x}$ and $10 \mathrm{x}$ objective) which were subcultured (48 h) onto CCG agar. CCG broth cultures were incubated for 1-3 weeks with shaking (120 strokes/min; $\left.28^{\circ} \mathrm{C}\right)$.

\section{Characterization and identification of the isolate QUBC97}

Streptomyces isolate color was observed on ISP4 (BD Difco) [9]. Microscopic examination at 4x, 10x, and 45x objectives of isolate QUBC97, documented with Exilim camera, Casio, Japan). The guide for identification of Streptomyces sp. was consulted [10]. 
Several loci, 16S, superoxide dismutase (SOX), and amylase were employed in the identification of QUBC97 in addition to morphological features. The loci, primers, and PCR product size are detailed (Table 2) [11,12]. Sequenced amplicons and multilocus analyses were employed to include or exclude QUBC97 from lists generated by primer nucleotide-BLAST.

\begin{tabular}{|c|c|c|c|}
\hline Primre & Sequence & Tm & Reference \\
\hline SMycF/16S & 5'-GGTCGAAAGCTCCGGCGGTGAA-3' & 60 & 11 \\
\hline SMycF2/16S & 5'-CCCTTCACTCTGGGACAAGCCC-3' & 61 & 11 \\
\hline SMycR/16S & 5'-GAGTCCCCAACACCCCGAAGG-3' & 60 & 11 \\
\hline QUGPRn3/16S & 5'-GGCGTGGACTACCAGGGTATC-3' & 65 & 13 \\
\hline QUGP Fn5/16S & 5'-ACTCCTACGGGAGGCAGCAG-3' & 65 & 13 \\
\hline QUGP Fn6/16S & 5'-CCAGCAGCCGCGGTAATAC-3' & 62 & 13 \\
\hline QUGPRn2/16S & 5'-TGACGGGCGGTGTGTACAAG-3' & 63 & 13 \\
\hline $\begin{array}{c}\text { SOX F (Superoxide } \\
\text { dismutase) }\end{array}$ & 5'-GCAGGTAGAAGGCGTGCTCCCA-3' & 62 & 13 \\
\hline SOX R & 5'-CCTGGCCTTCCACCTCTCCGG- & 61 & 11 \\
\hline Amylase F & 5'-GTGGTGGACCTCCTACCAGCC-3' & 61 & 11 \\
\hline Amylase R & 5'-CGTCGATGCGGAAGCCGTC-3' & 61 & 11 \\
\hline
\end{tabular}

${ }^{*}$ PCR product size SMycR with SMycF2, 1005; with SMycF, 934; with QUGPFn5, 800; QUGPFn6, 640bp. Also, QUGPRn2 with SMycF2,1265; with SMycF, 1172bp. If QUGPRn3 instead of QUGPRn2, PCR product is 595bp shorter. QUGP;Al-Quds University General Primers [13]

Table 2: Primers used in this work

Protocol of PCR amplification was: $95^{\circ} \mathrm{C}$ for $2 \mathrm{~min}$, followed by 31 cycles of denaturation at $94{ }^{\circ} \mathrm{C}$ for $90 \mathrm{~s}$, annealing for $30 \mathrm{~s}$ at 60 ${ }^{\circ} \mathrm{C}, 30 \mathrm{~s}$ at $58^{\circ} \mathrm{C}$, and extension at $72{ }^{\circ} \mathrm{C}$ for $105 \mathrm{~s}$. A final extension at $72{ }^{\circ} \mathrm{C}$ for $2 \mathrm{~min}$. PCR was carried out using Master Mix from Promega Biochemical Company, products were analysed on $1.6 \%$ agarose containing $1 \mu \mathrm{g} / \mathrm{ml}$ ethidium bromide in Tris-acetateEDTA (TAE) buffer, pH 8.

Selected amplicons were extracted using NeucleoTrap, Mecherey Nagel, Germany and sequenced (Bethlehem University, Bethlehem, Palestine) then analysed by BLAST nucleotide alignment.

\section{Antibacterial bioassay tests}

Three simple screening tests were devised and applied in antibiotic evaluation.

Agar plug diffusion test: Four-week old Streptomyces CCG agar plates $\left(28^{\circ} \mathrm{C}\right)$ were used to obtain long agar plugs using a sterile scalpel blade or the wide end of Pasteur pipette kept in $70 \%$ alcohol, drained, flamed, cooled, and used to cut agar plugs near growth areas. Agar plugs were placed on lawns of target bacteria/yeast (Bacillus atrophaeus QUBC16, baker's yeast Sacchromyces cerevisoae, or other bacterial isolates) prepared on nutrient agar (NA) plates $[13,14]$. After overnight incubation at $30{ }^{\circ} \mathrm{C}$, zones of inhibition were observed. Repetition was done to confirm results.

n-butanol extraction: One $\mathrm{ml}$ aliquot was collected from 50-ml liquid CCG culture grown in 150 ml-Erlenmeyer flasks; shaking at 120 strokes/min at $28^{\circ} \mathrm{C}$ for indicated time, cleared by centrifugation. The supernatant was vigorously mixed with $0.3 \mathrm{ml} \mathrm{n}$-butanol and briefly centrifuged. Ten $\mu \mathrm{l}$ of butanol top layer were placed on 3-mm sterile paper discs, air dried, and placed on bacterial lawns prepared on NA plates, incubated at $30^{\circ} \mathrm{C}$ and inspected after overnight incubation for zones of inhibition.

This method was used to study the kinetics of antibacterial production; Three filter paper discs were impregnated each with $10 \mu \mathrm{l}$ n-butanol extracted daily for 7 days, allowed to air-dry, and placed on the surface of NA coated with Bacillus atrophaeus QUBC16 [13]. Diameters of zones of inhibition were measured to reflect productivity.

Large scale extraction of antimicrobial activity of Streptomyces QUBC97, two $750 \mathrm{ml}$ CCG medium each placed in 2-liter flasks with shaking ( 120 Strokes $/ \mathrm{min}$ ) for 2 weeks at $28^{\circ} \mathrm{C}$, centrifuged in $30-\mathrm{ml}$ tubes at $25,000 \mathrm{rpm}$ for 15 min, supernatant was pooled in a separatory funnel, mixed vigorously with n-butanol $(4: 1 \mathrm{v} / \mathrm{v})$ respectively, allowed to settle overnight, and the upper layer was collected. A second extraction was performed similarly. The two n-butanol fractions were pooled and evaluated by the disc diffusion method. The extract labelled as crude n-butanol extract ( $\mathrm{cnBE}$ ). Using rotary evaporator at $50{ }^{\circ} \mathrm{C}$, $\mathrm{cnBE}$ was concentrated to near dryness.

Evaluation of antimicrobial agent mobility by thin layer chromatography (TLC): Ten $\mu$ l of cnBE from broth or agar plates were spotted on the base line of silica thin layer chromatography plate strips $(1 \times 8 \mathrm{~cm})$, allowed to air-dry and developed in different solvents. Dried TLC strips were visualized under UV light, and/or placed face down on lawns of target organism on NA plates, after overnight incubation at $30^{\circ} \mathrm{C}$ plates were examined for presence of zones of growth inhibition.

Solvent systems used to develop the TLC strips were: The top layer of n-butanol equilibrated with 1.7\% ammonium acetate (nBA) [15]. Other solvent systems were prepared according to HEMW system; Hexane, Ethyl acetate, Methanol, and Water [16]. 


\section{HPLC of concentrated crude n-butanol extract (cnBE)}

Concentrated cnBE (n-butanol extraction section) was dissolved in water and subjected to preparative HPLC purification. Four peaks wear eluted in water, only one peak showed antibacterial activity as evaluated by disc diffusion method. The two positive fractions $(50 \mathrm{ml}$ each) were pooled and re-extracted with $25 \mathrm{ml} \mathrm{n}$-butanol from the aqueous HPLC column fractions and concentrated by rotary evaporation at $50{ }^{\circ} \mathrm{C}$. The dried material was re-suspended in $1 \mathrm{ml}$ autoclaved pure water for analysis by mass-spectroscopy for putative identification.

\section{Inhibitory effect of QUBC97 cnBE extract against bacteria}

Ten $\mu$ l-discs were air-dried (n-butanol extraction section), diffusion method was used against a number of bacterial species: Staphylococcus epidermidis, several S. aureus isolates, Streptococcus viridians, S. pyogenes, S. aglactiae, Bacillus atrophaeus, Escherichia coli (several isolates), E. coli HB101 laboratory strain, and several isolates of Pseudomonas aeruginosa. The cnBE extract was tested against three clinical Methicillin Resistant S. aureus (MRSA) and baker's yeast as well.

\section{Results}

\section{Isolation and maintenance of Streptomyces species}

Tens of Streptomyces spp. were simply obtained from soil samples on CCG medium. QUBC97 was initially isolated on nutrient agar and maintained on CCG medium. Microscopy allowed quick subculturing of microscopic colonies that were identified by direct microscopic ( $4 \times$ and $10 \times$ objectives) examination of CCG plates.

Putative identification of the aerobic colonies showing fine microscopic filamentous mycelia, and compact colonies with or without powdery spores were essential features that putatively identified Streptomyces colonies. ISP4 and CCG cultures were used for further macroscopic and chemical characterization (Figure 1).

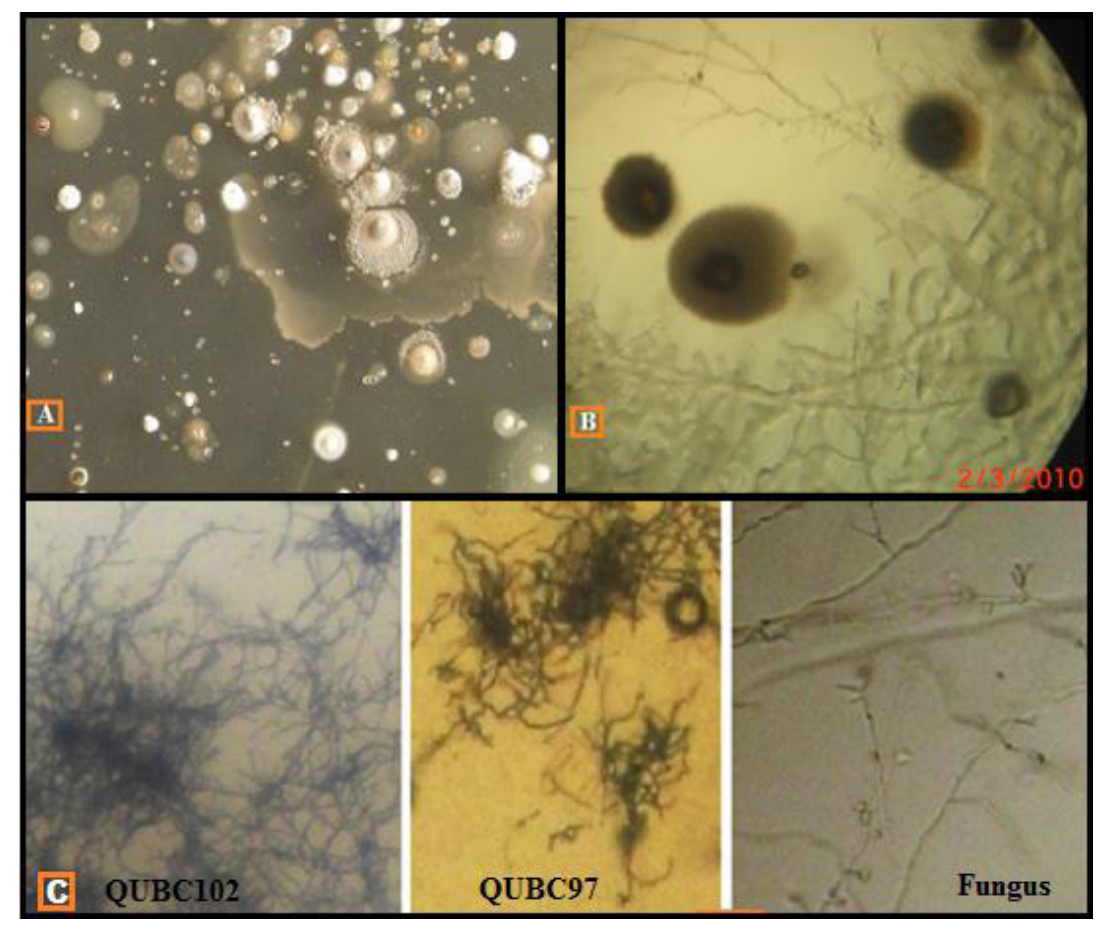

Figure 1: A. Primary soil sample cultured on CCG medium. B. Three-day old soil CCG culture showing fungus and streptomyces growth (4x objective view). C. Isolated Streptomyces QUBC102 and QUBC97 compact and thin mycelia versus thick fungal mycelia (10x objective) as seen through CCG agar medium.

\section{Streptomyces QUBC97 and other isolates}

Screening several isolates with the Agar Plug Diffusion assay, QUBC97 was selected for further investigation. Colonies of isolate QUBC97 showed white areal powdery mycelia and yellow growth on both CCG Figure (2A) and ISP4 Figure (2C). Diffused yellow product on CCG Figure (2B) or ISP4 Figure (2D). The opaque nature of ISP4 due to its starch and calcium chloride content dimed the intensity of yellow pigmentation relative to the transparent CCG agar. The low complexity of CCG medium allowed studying antimicrobial productivity and agent extraction in relatively pure form.

\section{Testing for antimicrobial activity}

\section{Agar plug diffusion test}

Agar plugs were cut out from CCG agar plates after 14 days from areas near heavy growth of pure cultures of putative Streptomyces 
isolates (Figure 2), antibiotic diffusion agar plugs were used as in filter paper disc sensitivity tests. Regardless of shape of agar plugs, agar plugs were placed on Bacillus atrophaeus QUBC16 lawns prepared on nutrient agar and incubated at $30{ }^{\circ} \mathrm{C}$. Figure 3 shows QUBC97 and two other isolates that produced anti-bacillus agents. QUBC97 was selected for further investigation.

\section{QUBC97 activity against clinical isolates}

Figure 4 shows zones of inhibition generated by crude n-butanol extract ( $10 \mu \mathrm{l}$ cnBE per disc) from QUBC97 culture. Inhibition of Gram positive Bacillus atrophaeus QUBC16, Staphylococcus epidermidis, Staphylococcus aureus, Streptococcus pyogenes, Streptococcus aglactiae in addition to Bacillus subtilis QUBC18 and other Bacillus spp. (not shown). Also it was effective against Gram negative bacteria; E. coli HB101, a clinical Pseudomonas aeruginosa 1, and three clinical MRSA. Resistant bacteria included: Bacillus sp. QUBC142 (Figure 3), Escherichia coli DH5a, clinical P. aeruginosa 7, and baker's yeast.

\section{Kinetics of antibiotic production}

Longitudinal sampling of liquid culture medium every 24 hours for seven days followed by immediate extraction with n-butanol, and disc diffusion assay against B. atrophaeus QUBC16 revealed varying zones of inhibition. Diameter of zone of inhibition was viewed as a direct reflection of the concentration of antibacterial agent. For each time point 3 readings were obtained. Diameters of zones of inhibition were measured after $24 \mathrm{~h}$ incubation at $28^{\circ} \mathrm{C}$ (Figure 5).
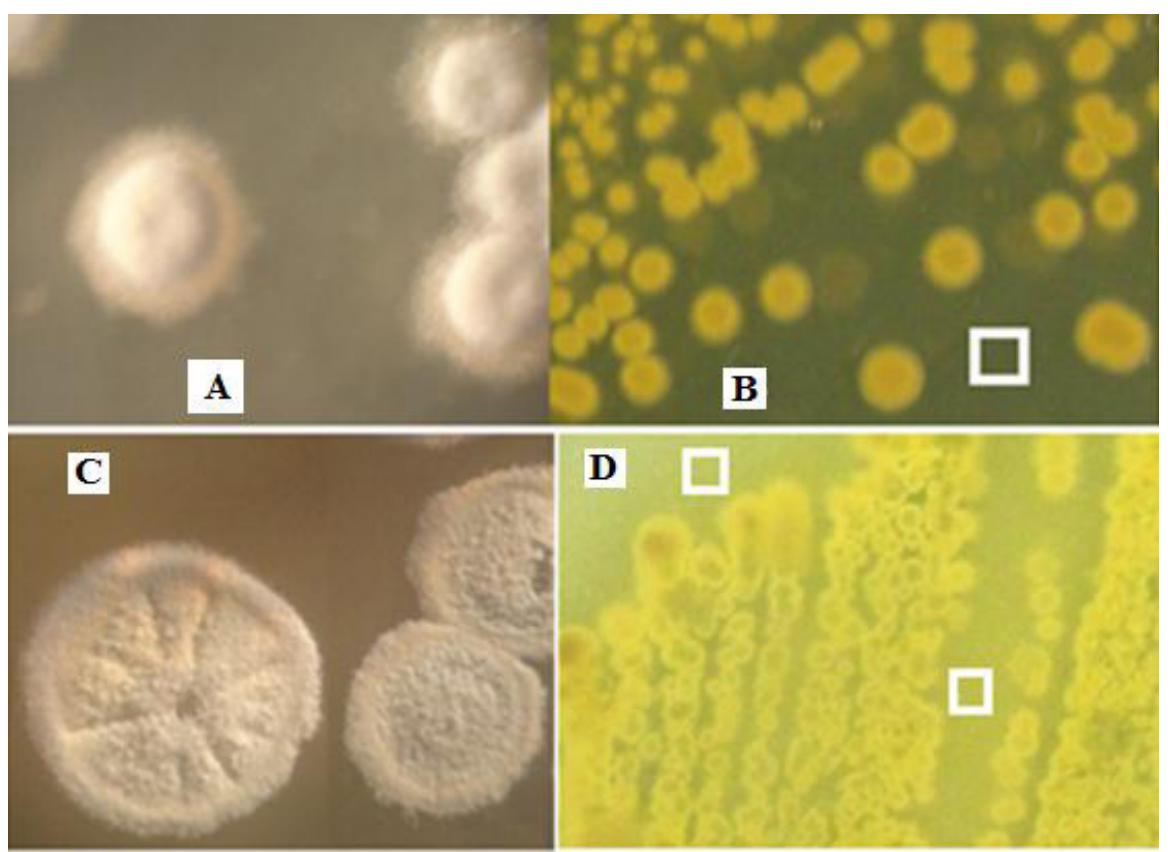

Figure 2: Isolate QUBC97, white areal powdery colonies on CCG agar (A) or ISP4(C), yellow growth pigmentation of the lower side of colony and diffused yellow product on CCG (B) or ISP4 (D). White square (B) indicate candidate areas for obtaining agar plugs used in antimicrobial screening

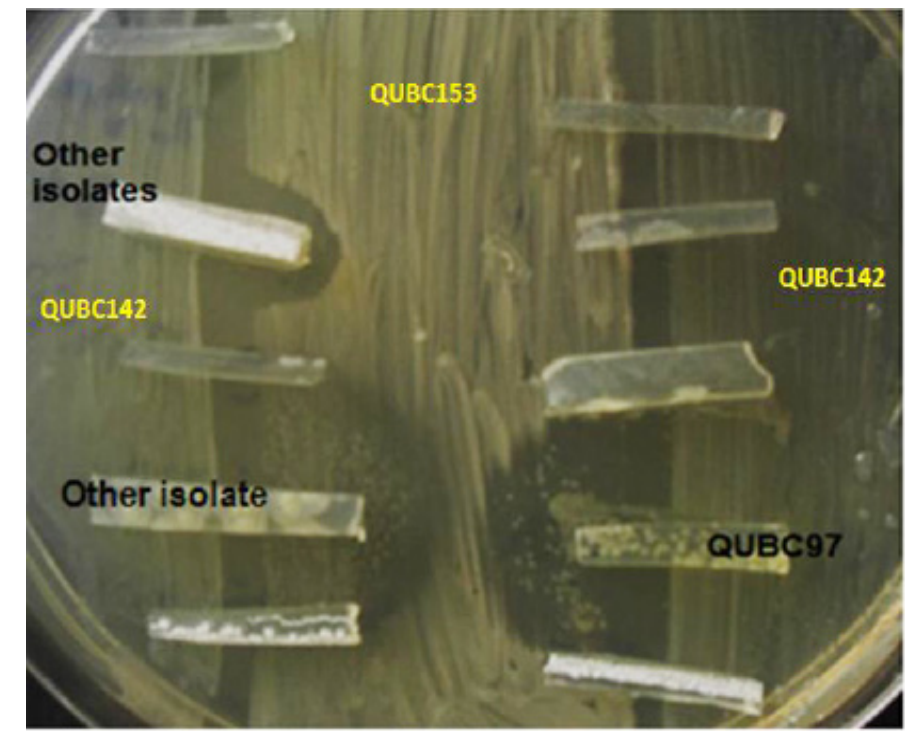

Figure 3: Screening for antibacterial activity. QUBC97 among three different Streptomyces isolates showing inhibition zones around agar plugs, against Bacillus sp. QUBC153. Other isolates were not effective, whereas Bacillus sp. QUBC142 was not responsive to any agar plug 

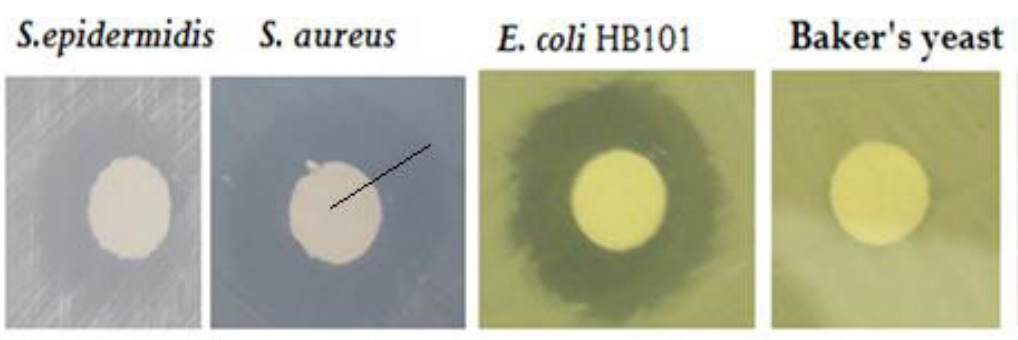

\section{P. aeruginosa}
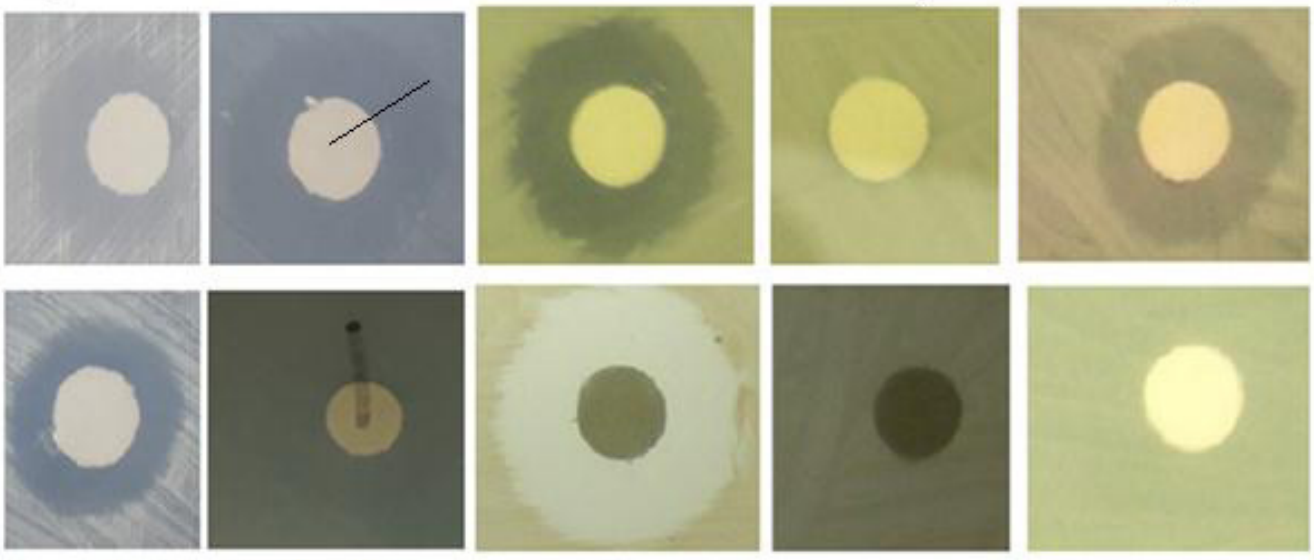

S. agalactiae S. pyogenes

B. atrophaeus P. aeruginosa 7

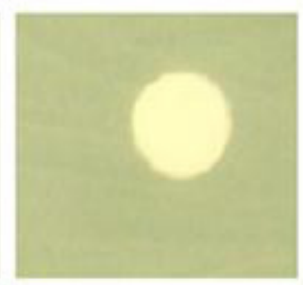

Effect of $10 \mu \mathrm{l}$-Butanol extract of QUBC97 Culture

Figure 4: Ten $\mu \mathrm{l}$ of n-butanol extracted QUBC97 culture were loaded on paper discs and allowed to air-dry before being placed on bacterial lawns. Activity of $\mathrm{n}$-butanol antibiotic against different microorganisms, using impregnated filter disc diffusion technique. Lines indicate zones of inhibition for S. aureus and Streptococcus pyogenes. The cnBE was also active against three clinical Methicillin Resistant S. aureus (MRSA)

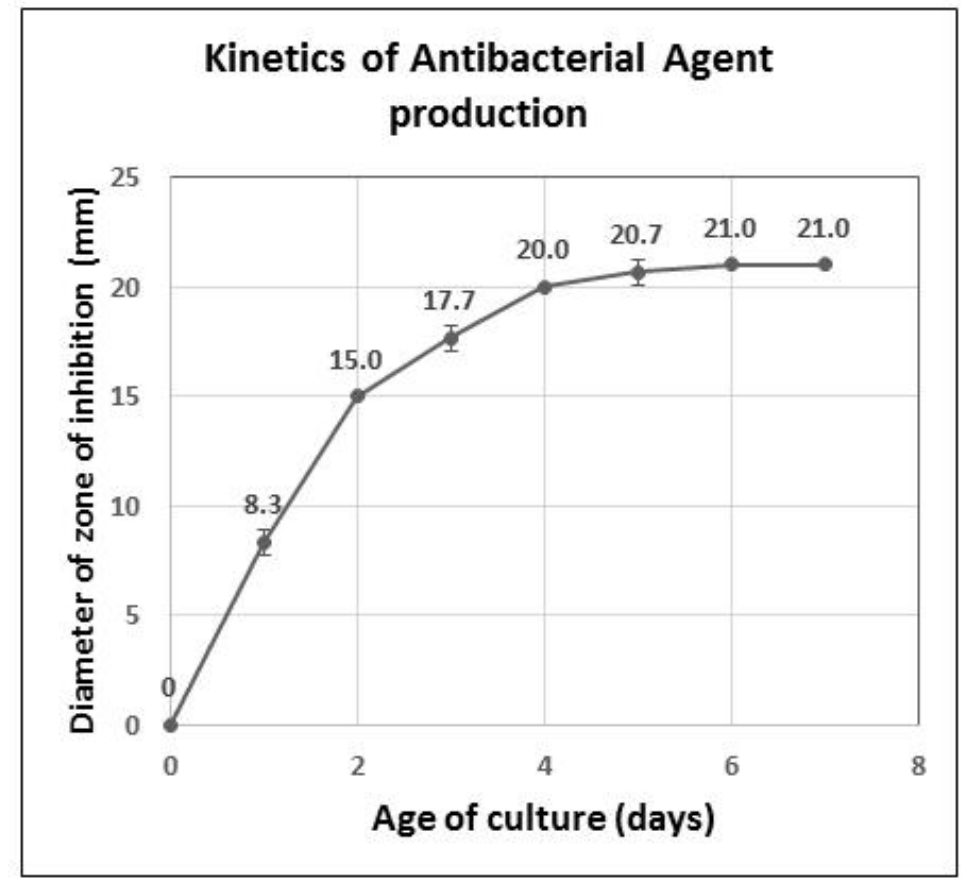

\begin{tabular}{|l|l|l|l|l|l|l|l|}
\hline QUBC97 culture age (days) & 1 & 2 & 3 & 4 & 5 & 6 & 7 \\
\hline Zone of inhibition in mm (n=3) & 8.3 & 15 & 17.7 & 20 & 20.7 & 21 & 21 \\
\hline Standard deviation (SD) & 0.57 & 0 & 0.57 & 0 & 0.57 & 0 & 0 \\
\hline
\end{tabular}

Figure 5: Kinetics of antibacterial production by QUBC97. n-Butanol extract (10 $\mu$ l) were placed on $3 \mathrm{~mm}$ filter disc and allowed to air dry and tested against Bacillus atrophaeus QUBC16; $\mathrm{n}=3$. Maximum production under indicated conditions can be achieved after $\sim 130 \mathrm{~h}$ where it stabilized at $21 \mathrm{~mm}$. Error bars indicate standard deviation from the mean

The production of the antibiotic appeared one day post incubation and increased with time and reached its maximum level after 6 days of incubation as reflected by the $21-\mathrm{mm}$ zone of inhibition.

\section{TLC analysis of butanol extract}

The recovered n-butanol extract from agar plates of QUBC97 was analyzed by thin layer chromatography on silica gel plates (TLC). The results showed that two compounds can be separated into two bioactive spots when TLC was developed in n-butanol saturated with $1.7 \%$ ammonium acetate (nBA); $\mathrm{R} f$ - values were 0.62 and 0.85 (Figure 6).

In another solvent (water: methanol; $1: 1 \mathrm{v} / \mathrm{v}$ ), $\mathrm{R} f$ was 0.17 and another product appearing as a streak along the TLC strip as indicated by the zone of inhibition along the TLC plate (Figure 6). 


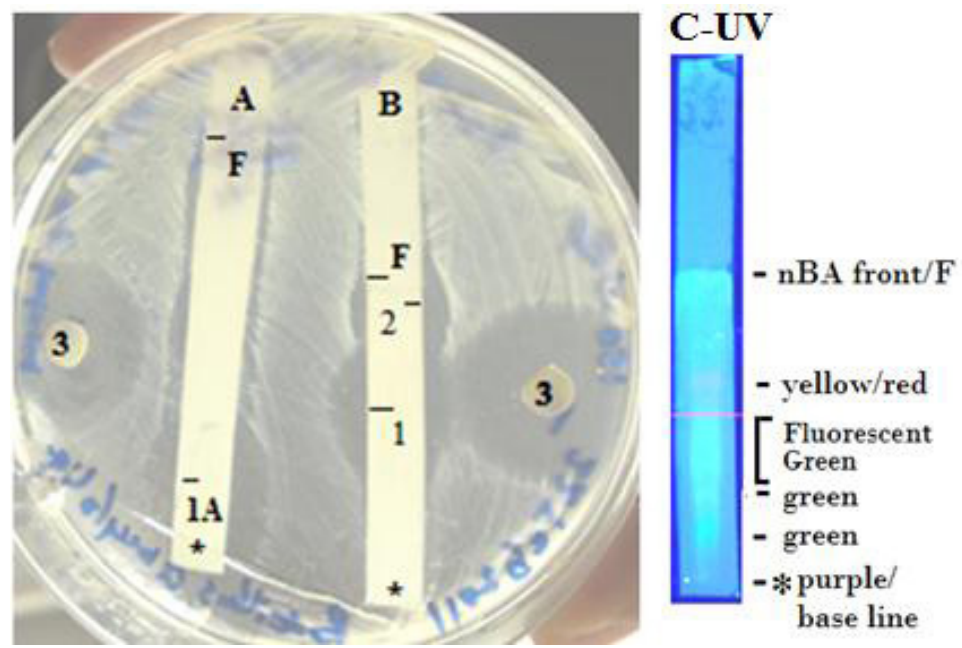

Figure 6: Agar plate of QUBC97 grown on CCG extracted with n-butanol. A: TLC developed in methanol: water (1:1), spot $1 \mathrm{~A}$ R $f=0.17$ and a long streak of inhibition. B: that was developed in $\mathrm{nBA}$; spot 1 with $\mathrm{R} f=0.62$ and spot $2 \mathrm{R} f=0.84 ; \mathrm{F}$ is the solvent front. The disc (3) diffusion test showing a diameter of $22 \mathrm{~mm}$ of $5 \mu \mathrm{l}$ extract. TLC plate (C-UV photo) showed an orange/yellow spot that coincides with the slow moving activity at $22 \mathrm{~mm}$ (B, spot 1 ) from TLC base line ${ }^{*}$ ) suggesting the yellow diffused substance to be the active component or that co-purifies with the active component

When extracts were obtained from liquid QUBC97-cultures, only one (fresh FCm or old OCm) product could be extracted with nbutanol depending on the age of culture. Therefore, liquid media provided a simple method to obtaining one or the other product for further analysis. FCm and OCm products prepared separately, when co-spotted on the same TLC plate they showed different $\mathrm{R} f$-values (Figure 7).

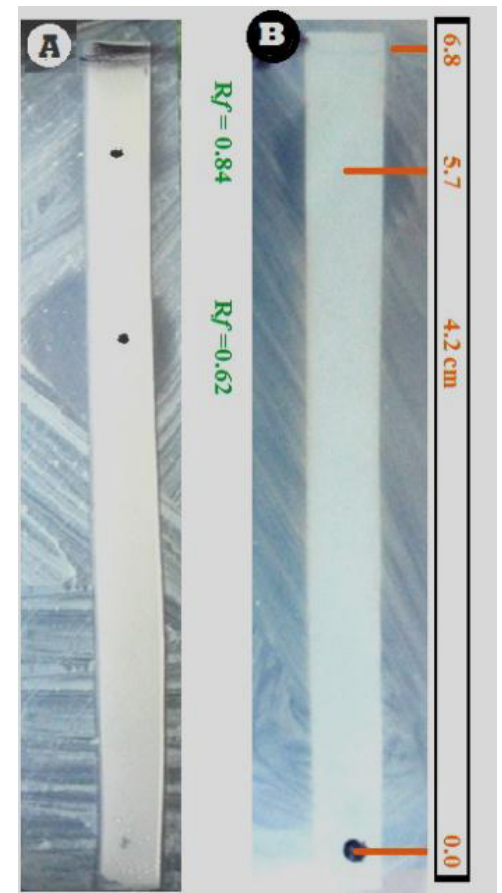

Figure 7: Fresh and old n-butanol extracts were mixed on the same TLC (A). Fresh (B) or old (not shown) were spotted separately. TLCs were developed in $\mathrm{n}$-Butanol/Ammonium acetate. $\mathrm{Rf}=0.84$ of fresh compound $(\mathbf{A} \& \mathbf{B})$. Old culture appeared at $\mathrm{Rf}=0.62$. Consistent with Figure 6

The n-butanol extract of liquid medium (FCm) showed an $\mathrm{R} f$-value of 0.3 when developed in a polar solvent (+6: a mixture of Hexane: Ethylacetate: methanol: water; 2:8:2:8). Better mobility $(\mathrm{R} f=0.72)$ was obtained when TLC were developed in aqueous phase of a less polar solvent (-2; Hexane: Ethylacetate: methanol: water; 7:3:5:5) [16].

Visible light absorption of the slower compound (OCm sample; $\mathrm{R} f=0.62$; $\mathrm{nBA}$ solvent) showed a split absorption peak at $428 \mathrm{~nm}$ and $442 \mathrm{~nm}$. Similarly, the faster (FCm; $\mathrm{R} f=0.84 ; \mathrm{nBA}$ solvent) showed a split peak at $426 \mathrm{~nm}$ and $442 \mathrm{~nm}$.

\section{Chemical nature of the n-butanol extracted antibacterial agent}

MS analysis of the HPLC purified sample suggested the chemical formula $\mathrm{C}_{8} \mathrm{H}_{9} \mathrm{NO}_{2}$, identified with $80 \%$ probability as methoxyphenyl-oxime (Figure 8A). Probability of being 4-ethylbenzoic acid, 2-butyl ester probability was 6.84\%; and 4-ethylbenzoic acid, cyclopentyl ester $6.04 \%$. The MS spectrum of the compound was different from that of ethanone, 1-phnyl-, oxime. The n-butanol 
extract remained bioactive after 3 years of preparation and storage at room temperature. Changing the pH of agar plugs by soaking in sodium hydroxide or hydrochloric acid appeared to increase the zone of inhibition (Figure 8B).

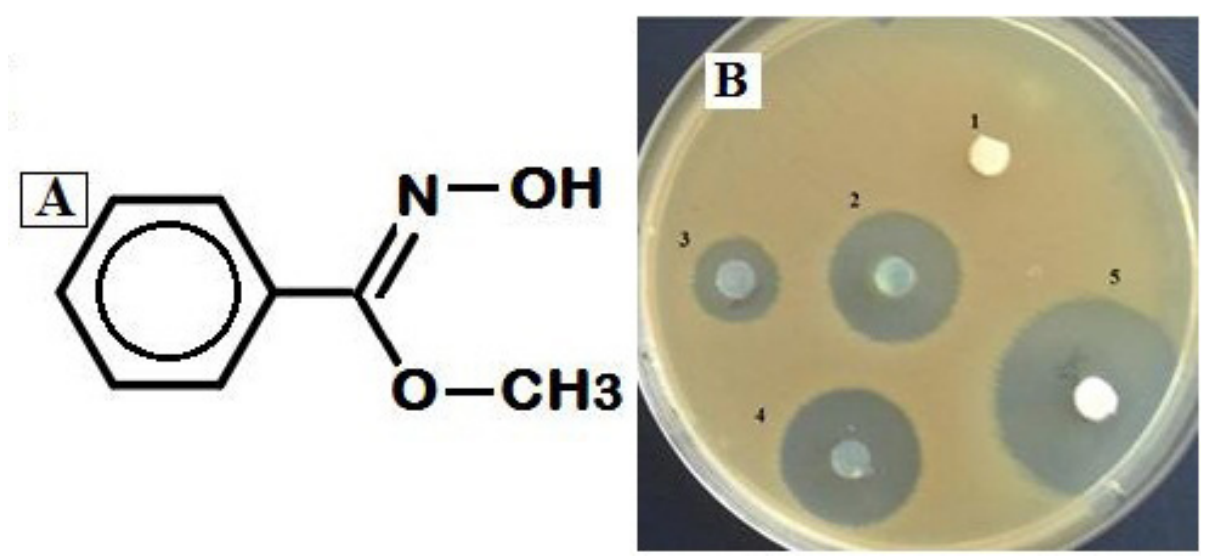

Figure 8. A: Mass spectrometry analysis of HPLC purified agent. $\mathrm{m} / \mathrm{s}$ peaks based on relative intensity from high to low: 133, 151, $135,134,152,77,68,75,207,281$ identified a chemical compound with the formula C8H9NO2 as methoxyphenyl-oxime. B- Effect of

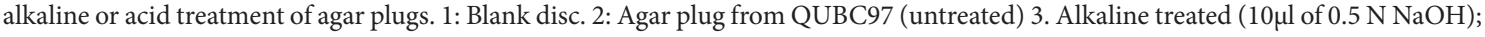
4, Acid treated $(5 \mu \mathrm{l}$ of $3 \mathrm{~N} \mathrm{HCl})$. Disc 5 contained $5 \mu$ each of fresh and old compounds.

\section{Identification of QUBC97 Streptomyces species}

Figure 9 shows PCR amplicons of superoxide dismutase (SOX), amylase amplicon, and a number of 16S amplicons, each amplicon was generated from QUBC97 with Streptomyces general primers. BLAST analysis of 502-nucleotide sequence of the 16S amplicon (Table 3) matched 17 Streptomyces spp. with 100\% coverage and 99\% identity (Table 4) including most of the S. pratensis phylogroup [5]. BLAST results of superoxide dismutase (SOX: 329 b) sequence (Table 3) returned 190 matches with $99 \%$ identity. Common species that matched both SOX and 16S identified QUBC97 as Streptomyces pratensis ATCC 33331, S. microflavus, or S. fulvissimus. Since QUBC97 produced positive amylase PCR reaction (386bp), S. microflavus was eliminated because it produced a negative amylase BLAST result. It also was negative when BLAST-analyzed with amylase primers (Table 2). S. fulvissimus and S. pratensis shared amylase amplicon and could not be excluded. However, both S. microflavus and S. fulvissimus unlike QUBC97 do not produce diffusible pigments on organic media [10]. Analyses suggested that QUBC97 was most likely related to $S$. pratensis phylogroup [5].

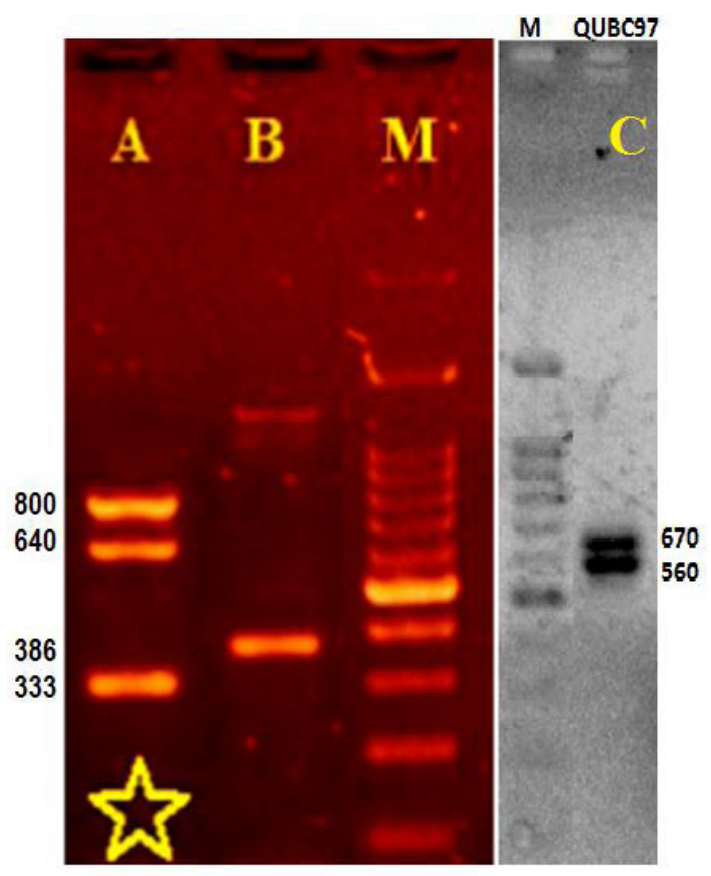

Figure 9: Multilocus PCR. Agarose Gel (1.6\%) showing multiple PCR amplicons from isolate QUBC97; lane A: 16S ribosomal amplicons (800 and $640 \mathrm{bp}$ ) and superoxide dismutase $333 \mathrm{bp}$ (asterisk; sequenced). Lane B, 16S 1265 (SMycF2 SMycR); and 16S 1172 (faint band SMycF SMycR), amylase amplicon at 386 bp. Lane C, 16S generated with SMycF and QUGPRn3 (670bp sequenced) and SMycF2.Rn3(560bp). 


\begin{tabular}{|l|l|}
\hline 16S: \\
\hline AAAACTCCGGCGGTGAAGGATGAGCCCGCGGCCTATCAGCTTGTTGGTGGGGTAATGGCCTACCA \\
AGGCGACGACGGGTAGCCGGCCTGAGAGGGCGACCGGCCACACTGGGACTGAGACACGGCCCAG \\
ACTCCTACGGGAGGCAGCAGTGGGGAATATTGCACAATGGGCGAAAGCCTGATGCAGCGACGCCG \\
CGTGAGGGATGACGGCCTTCGGGTTGTAAACCTCTTTCAGCAGGGAAGAAGCGAAAGTGACGGTA \\
CCTGCAGAAGAAGCGCCGGCTAACTACGTGCCAGCAGCCGCGGTAATACGTAGGGCGCAAGCGTT \\
GTCCGGAATTATTGGGCGTAAAGAGCTCGTAGGCGGCTTGTCACGTCGGATGTGAAAGCCCGGGG \\
CTTAACCCCGGGTCTGCATTCGATACGGGCTAGCTAGAGTGTGGTAGGGGAGATCGGAATTCCTGG \\
TGTAGCGGTGAAATGCGCAGATATCAGGAGGAACACCGGTGGCGAAGGCGGATCTCTGGGCCATT \\
ACTGACGCTGAGGAGCGAAAGCGTGGGGAGCGAACAGGATTAGATACCCTGGTAGTCCACGCCGT \\
AAACGTTGGGAACTAGGTGTTGGCGACATTCCACGTCGTCGGTGCCGCAGCTAACGCATTAAGTTC \\
CCCGCCTGGGGAGTACGGCCGCAAGGCTAAAACTCAAAGGAATTGACGGGGGCCCGCACAAGCA \\
GCGGAGCATGTGGCTTAATTCGACGCA \\
\hline SOX (267b) \\
\hline CCTGGCCGACGTTGCCCTGGTGGTCGTAGACCTGCTCGACGATCAGCTTGCCGCTCACGGGCTCGT \\
ACGCGAGCACGCCCCAGCCGGAGCCCTGGGTGGTGGCGGCGGCCTTCGTCAGCTGGGACTTGAAG \\
CCCGCGTAGGAGCCGAAGGACTCGGTGATCGCGTCGGCGAGGTCGCCCACGCCGTCCGCCGCGAG \\
GGGCTCGCCGCCGCCGTCGCCGGTCATGTTGTGCCAGTAGATCGAGTGCAGGATGTGGCCGGAGA \\
GGTGGA
\end{tabular}

742 base 16S DNA sequence and SOX from QUBC97 results Table 3: Produced DNA sequences

\begin{tabular}{|c|c|c|c|c|c|}
\hline & Streptomyces Species ${ }^{\star}$ & Score & $\%$ Coverage & \% Identity & Accession \\
\hline 1 & S. acrimycini strain CSSP430 & 1635 & 100 & 99 & NR_115449.1 \\
\hline 2 & S. alboviridis strain CSSP 419 & 1635 & 100 & 99 & NR_115374.1 \\
\hline 3 & S. anulatus strain NBRC 12755 & 1635 & 100 & 99 & NR_041062.1 \\
\hline 4 & S. baarnensis strain NBRC 14727 & 1635 & 100 & 99 & NR_112440.1 \\
\hline 5 & S. caviscabies strain ATCC 51928 & 1635 & 100 & 99 & NR_114493.1 \\
\hline 6 & S. cyaneofuscatus strain CSSP436 & 1635 & 100 & 99 & NR_115383.1 \\
\hline 7 & S. fimicarius strain CSSP537 & 1635 & 100 & 99 & NR_043351.1 \\
\hline 8 & S. flavofuscus strain NBRC 100768 & 1635 & 100 & 99 & NR_112591.1 \\
\hline 9 & S. flavogriseus strain ATCC 33331 ** & 1635 & 100 & 99 & NR_074559.1 \\
\hline 10 & S. praecox strain CSSP720 & 1635 & 100 & 99 & NR_115437.1 \\
\hline 11 & S. fulvorobeus strain NBRC 15897 & 1635 & 100 & 99 & NR_041196.1 \\
\hline 12 & S. griseoplanus strain CSSP 437 & 1635 & 100 & 99 & NR_043377.1 \\
\hline 13 & S. microflavus strain NBRC 13062 & 1635 & 100 & 99 & NR_112354.1 \\
\hline 14 & S. fulvissimus strain DSM 40593 & 1635 & 100 & 99 & NR_103947.1 \\
\hline 15 & S. pratensis strain ch $244^{\star *}$ & 1635 & 100 & 99 & NR_125616.1 \\
\hline
\end{tabular}

*Species (not strains) matching those reported by Rong et al [5] as S. pratensis ATCC33331 phylogroup are shown in bold letters; ${ }^{* *}$ matched both species and strain. All species were eliminated based on Superoxide dismutase sequence except for S. microflavus strain NBRC 13062, S. fulvissimus strain DSM 40593, and S. pratensis strain ch24 (lines 13, 14, and 15) Table 4: List of BLAST results

16S sequence (742b) from QUBC97 that only produced 100\% sequence coverage and 99\% identity (Maximum score; 1637)

\section{Discussion}

This work contributed the formulation and application of the new Citrate-Casamino-Glycerol (CCG) medium as a medium for physiological manipulations and by-product analyses. Tens of confirmed Streptomyces isolates were obtained [11-13] in the absence of cyclohexamide, nalidixic acid, or nystatine [18]. The selectivity of GCC medium may be related to its alkaline pH ( 7.8 before autoclaving) and poor nutritional content. The transparency of the medium allowed direct microscopic examination and enabled putative identification of Streptomyces (Figure 1). Simple media are preferred for assaying antibiotic production; glycerol is used by several Streptomyces spp. [19,20]. Agar Plugs used in antibiotic screening were cut from three-week old Streptomyces CCG agar cultures (Figure 3) and were effectively used in presumptive evaluation of antimicrobial activity.

Integration of molecular and morphological methods appeared to simplify Streptomyces species identification which is not a simple task [5]. Superoxide dismutase (SOX) and 16S sequencing, amylase PCR and morphological characteristics identified Streptomyces QUBC97 as a member of the Streptomyces pratensis phylogroup, a results that is in agreement with Rong et al report [5].

QUBC97 matched 7 of the species placed in the S. pratensis phylogroup based on 16S sequences and matched the strains $S$. pratensis ATCC33331 and S. griseus NBRC 13350 of the same 16S phylogroup [5]. Yet genome BLAST alignment of Streptomyces flavogriseus strain ATCC 33331 (Streptomyces flavogriseus IAF-45-CD =ATCC 33331 recently reclassified as S. pratensis ATCC33331) 
[1], revealed S. griseus NBRC 13350 as the closest match. Only 58\% genome coverage and 88\% identity ( $\mathrm{Ti}=51 \%)$ whereas $\mathrm{Ti}$ was near 100\% based on $16 \mathrm{~S}$ matching. Although QUBC97 was closely related to Streptomyces pratensis ATCC 33331. Despite some discrepancies that separated QUBC97 from S. pratensis ch24, ATCC 33331, and other S. pratensis phylogroup species and strains; we identified the isolate as S. pratensis QUBC97.

This report is the first to show antibacterial activity of MPO from Streptomyces pratensis QUBC97, related oxime containing compounds other than MPO have been recovered from Streptomyces cultures and other sources [21-24]. Several synthetic oximes with antifungal properties have been reported [25]. Maize plant cytochrome P450 forms oximes from their aminoacids; phenylacetaldoxime and indole-3-acetaldoxime [26].

Chipps et al. reported on the cytotoxicity of MPO extracted as an aqueous component of bitter melon seed [27]. MPO is present as $4.34 \%$ fraction in ethanol extract of the plant Sedum pallidum; the crude extract showed antibacterial activity [28].

\section{Conclusion and Recommendation}

It is not clear how methoxyphenyl-oximes and other oximes behave as antibiotics. We have neither investigated whether MPO was bactericidal or bacteriostatic nor we have investigated its mechanism of action. Methoxyphenyl-oxime glycosides named Uzmaq-A and Uzmaq-B isolated from Aspergillus flavus AF612 are biosurfactants [29].

However, since MPO was not effective against some bacterial isolates and Baker's yeast we assumed it was not toxic and may have specific bacterial (prokaryotic target). MPO was effective against all three tested clinical isolates of Methicillin Resistant Staphylococcus aureus (MRSA) indicating it is separable from $\beta$-lactam antibiotics. Although MPO was effective against some Gram negative bacteria, others were resistant; these were E. coli DH5a but not HB101, Pseudomonas aeruginosa 1 but not P. aeruginosa 7 (Figure 4) suggesting that MPO may be a true specific antibacterial agent that controlled some bacteria but not others.

The appearance of another active antibacterial agent in old culture broth $(\mathrm{OCm})$ and disappearance of the fresh methoxyphenyloxime (FCm) was noticed. Both shared similarities; split peak light absorbance at 426/428 and $242 \mathrm{~nm}$ and anti-bacillus bioactivity. However, their mobility differed on TLC developed in nBA when co-spotted or when spotted individually, suggesting that one molecule was likely metabolized further to acquire a different form with different Rf value. TLC mobility of the compound in different solvents was consistent with MPO structure in terms of molecular polarity. When agar plugs were acid-or alkali-treated, increased zones of inhibition were observed, this enhancement may be due to increased agent solubility. Apparently, the acid/alkaline treatment did not cause degradation of the antibacterial function (Figure 8B). These characteristics of soluble diffusible yellow pigment that was extractable in n-butanol, with indicated Rf values, and light absorption at 426/428 and $442 \mathrm{~nm}$ may represent characteristic tags to $S$. pratensis QUBC97 or similar strains.

This QUB97 tag is similar to the intracellular or extracellular blue pigment tag produced by S. coelicolor A3(2) at different $\mathrm{pH}$ ranges described by Bystrykh et al. [30].

We recommend that researchers provide better description of their Streptomyces isolates since 16S DNA sequence must be coupled with other molecular, morphological, biochemical characteristics, or tags. Representative photos of colony color and morphology may aid others to compare strains and isolates.

The TLC bioassay system applied in this study (Figures 6 and 7) is a powerful tool towards preliminary characterization of the antibiotic agent, small scale purification, testing fractionated products from HPLC, extracts, or other preparation. It may provide an excellent means for comparative antibiotic studies.

\section{Acknowledgement}

Thanks to Al-Quds University and Dr. Ibrahim Abbassi, Al-Quds University for his suggestions and support. This work was presented as Master's Thesis [31].

We, the authors, declare that we have funded the presented work and we have no conflict of interest with any other parties.

\section{References}

1. Doroghazi JR, Buckley DH (2014) Intraspecies comparison of Streptomyces pratensis genomes reveals high levels of recombination and gene conservation between strains of disparate geographic origin. BMC Genomics 15: 970 .

2. Ghorbel S, Kammoun M, Soltana H, Nasri M, Hmidet N (2014) Streptomyces flavogriseus HS1: Isolation and Characterization of Extracellular Proteases and Their Compatibility with Laundry Detergents. BioMed Res Int 345980.

3. Taddei A, Rodríguez MJ, Márquez-Vilchez E, Castelli C (2006) Isolation and identification of Streptomyces spp. from Venezuelan soils: Morphological and biochemical studies. Microbio Res 161: 222-31.

4. Ju KS, Gao J, Doroghazi JR, Wang KK, Thibodeaux CJ, et al. (2015) Discovery of phosphonic acid natural products by mining the genomes of 10,000 actinomycetes. 4. Proc Natl Acad Sci USA 112: 12175-80

5. Rong X, Doroghazi JR, Cheng K, Zhang L, Buckley DH, et al. (2013) Classification of Streptomyces phylogroup pratensis (Doroghazi and Buckley, 2010) based on genetic and phenotypic evidence, and proposal of Streptomyces pratensis sp. System appl microbiol 36: 401-7. 
6. Berdy J (2005) Bioactive microbial metabolites. J Antibiot (Tokyo) 58: 1-26.

7. Cheng K, Rong X, Pinto-Tomás AA, Fernández-Villalobos M, Murillo-Cruz C, et al. (2015) Population Genetic Analysis of Streptomyces albidoflavus Reveals Habitat Barriers to Homologous Recombination in the Diversification of Streptomycetes. Appl Environ Microbiol 81: 966-75.

8. Gao B, Gupta RS (2012) Phylogenetic Framework and Molecular Signatures for the Main Clades of the Phylum Actinobacteria. Microbiol Mol Biol Rev 76: 66112 .

9. Shirling EB, Gottlieb D (1966) Methods for characterization of Streptomyces species. Int J Syst Evol Microbiol 16: 313-40

10. Waksman SA, Lechevalier HA (1953) Guide to the classification and Identification of the actinomycetes and their antibiotics. The Williams \& Wilkins Company, Baltimore, USA.

11. Qadi M and Barghouthi SA (2012) Multi-locus Typing of Streptomyces spp. The Seventh Palestinian Conference of Laboratory Medicine. Bethlehem, Palestine.

12. Barghouthi S, Ayesh M (2010) A semi-selective medium for the isolation and identification of Streptomyces spp. from soil samples. The Sixth Palestinian Conference of Laboratory Medicine. Al-Quds University.

13. Barghouthi S (2011) A Universal Method for the Identification of Bacteria Based on General PCR Primers. Indian J Microb 51: $430-44$.

14. Barghouthi S and Salman M (2010) Bacterial Inhibition of Orobanche aegyptiaca Pers. and Orobanche cernua Loefl. Radical Elongation. Biocontrol Sc Tech 20: 423-35.

15. Barghouthi S, Young R, Olson MOJ, Arceneaux JEL, Clem LW, et al. (1989) Amonabactin, a novel tryptophan- or phenylalanine-containing phenolate siderophore in Aeromonas hydrophila. J Bacteriol 171: 1811-16.

16. Friesen B, Pauli G (2016) Solvent Selection in Counter current Chromatography (CCC).

17. Barghouthi S, Abu-Lafi S, Ayesh M, Barghouthi S, Ghanem R, et al. (2010) Isolation and identification of Streptomyces spp. from soil samples. The Second Conference on Biotechnology Research and Applications in Palestine 26-27th September, 2010 An-Najah National University. Nablus, Palestine.

18. Khamna S, Yokota A, Peberdy JF, Lumyong S (2010) Indole-3-acetic acid production by Streptomyces sp. isolated from some Thai medicinal plant rhizosphere soils. EurAsia J BioSci 4: 23-32.

19. Zygmunt WA (1961) Oxytetracycline formation by Streptomyces rimosus in chemically defined media. Appl Microbio 9: 502-7.

20. Basak K, Majumdar SK (1973) Utilization of Carbon and Nitrogen Sources by Streptomyces kanamyceticus for Kanamycin Production. Antimicrob Agents Chemother 4: 6-10.

21. Silley P, Griffiths JW, Monsey D, Harris AM (1990) Mode of action of GR69153, a novel catechol-substituted cephalosporin, and its interaction with the tonB dependent iron transport system. Antimicrob Agents Chemother 34: 1806-8.

22. Wilson AM, Bailey PJ, Tasker PA, Turkington JR, Grant RA, et al. (2014) Solvent extraction: the coordination chemistry behind extractive metallurgy. Chem Soc Rev 43: 123-34.

23. Zhang W, Wei S, Zhang J, Wu W (2013) Antibacterial activity composition of the fermentation broth of Streptomyces djakartensis NW35. Molecules 8: 2763-8. 24. Kelly WL, Townsend CA (2005) Mutational Analysis of nocK and nocL in the Nocardicin A Producer Nocardia uniformis. J Bacteriol 187: 739-46.

25. Tu S, Xie YQ, Gui SZ, Ye LY, Huang ZL, et al. (2014) Synthesis and fungicidal activities of novel benzothiophene-substituted oxime ether strobilurins. Bioorg Med Chem Lett 24: 2173-6.

26. Irmisch S, Zeltner P, Handrick V, Gershenzon J, Köllner TG (2015) The maize cytochrome P450 CYP79A61 produces phenylacetaldoxime and indole-3-acetaldoxime in heterologous systems and might contribute to plant defense and auxin formation. BMC Plant Biology 15: 128-42.

27. Chipps ES, Jayini R, Ando S, Protzman AD, Muhi MZ, et al. (2012) Cytotoxicity analysis of active components in bitter melon (Momordica charantia) seed extracts using human embryonic kidney and colon tumor cells. Nat Prod Commun 7: 1203-8.

28. Dahpour AA, Rahdari P, Sobati Z (2012) Chemical composition of essential oil,antibacterial activity and brine shrimp lethality of ethanol extracts from Sedum pallidum. J Med Plants Res 6: 3105-9.

29. Ishaq U, Akram MS, Iqbal Z, Rafiq M, Akrem A, et al. (2015) Production and characterization of novel self-assembling biosurfactants from Aspergillus flavus. J App Microbiol 119: 1035-45.

30. Bystrykh LV, Fernández-Moreno MA, Herrema JK, Malpartida F, Hopwood DA, et al. (1996) Production of actinorhodin-related "blue pigmernts" by Streptomyces coelicolor A3(2). J Bacteriol 178: 2238-44.

31. Ayyad IMR (2016) Antimicrobial activity of a Number of Streptomyces species. M.Sc. Thesis, Al-Quds University, Jerusalem, Palestine

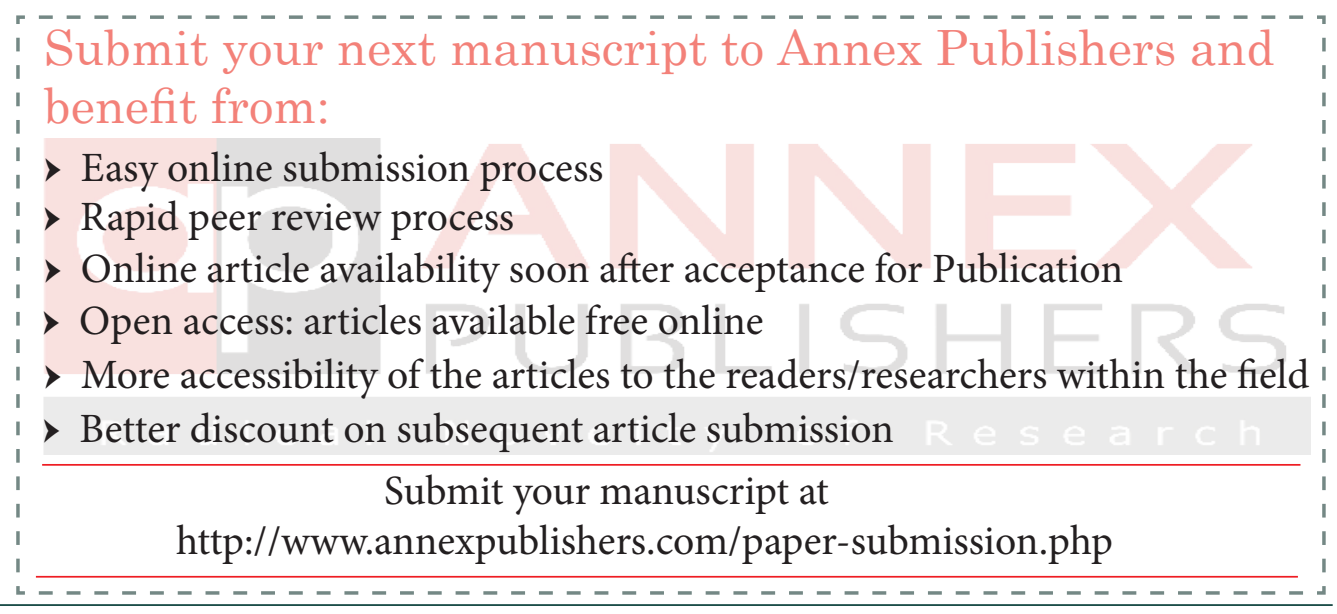

